\title{
DETERMINATION OF POLYMERIZ ATION PARTICLE MORPHOLOGY USING SYNCHROTRON COMPUTED MICROTOMOGRAPHY
}

K. W. Jones and P. Spanne

Brookhaven National Laboratory, Upton, New York 11973

W. B. Lindquist

State University of New York, Stony Brook, New York 11794

W. C. Conner and M. Ferrero

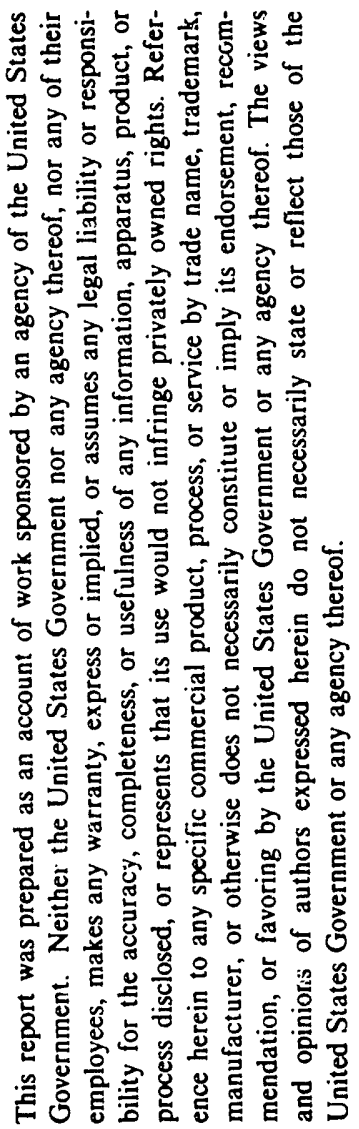

University of Massachusetts, Amherst, Massachusetts 00103

Presented at

Second International Conference on

Accelerators in Applied Research and Technology

Frankfurt am Main, Germany

September 3-7, 1991

By acceptance of this article, the publisher and/or recipient acknowledges the US Government's right to retain a nonexclusive, royalty-free license in and to any copyright covering this paper. 


\title{
DETERMINATION OF POLYMERIZATION PARTICLE MORPHOLOGY USING SYNCHROTRON COMPUTED MICRO'TOMOGRAPHY*
}

\author{
K. W. Jones and P. Spanne \\ Brookhaven National Laboratory, Upton, NY 11973 \\ W. B. Lindquist \\ State University of New York, Stony Brook 11794 \\ W. C. Conner and M. Ferrero \\ University of Massachusetts, Amherst, MA 00103
}

\begin{abstract}
Polymerization of monomers over heterogeneous catalysts results in the fragmentation of the catalysts and subsequent transport in the polymer particles that are produced. Characterization of the process using nondestructive synchrotron computed microtomography techniques makes possible measurement of the distribution of the catalyst fragments in an individual particle and, in addition, gives an estimate of the particle porosity and surface area. The present experiment was carried out using the $x$-ray microscopy facility at the Brooktaven National Synchrotron Light Source (NSLS) X26 beam line. The tomographic sections were analyzed using autocorrelation techniques to determine porosity and surface area values. The results are compared to values obtained using conventional methods. This prucedure makes possible the extraction of quantitative information about porosity and specific area from the tomograms.
\end{abstract}

Send correspondence to: Keith W. Jones

Building 815

Department of Applied Science

Brookhaven National Laboratory

Upton, NY 11973 USA

\footnotetext{
*Work supported in part by US Department of Energy Cantract No. DE-AC02-76CH00016 (KWJ,PS), NSF Grant CTS 89-21381 (WCC,MF), and by Applied Mathematics Subprogram of the US DOE Contract No. DE-FG02-90ER25084 (BL).
} 


\section{INTRODUCTION}

The polymerization of ethylene to produce polyethylene is accomplished using several types of supported catalysts. The initial size of the support particles is of the order of several hundred micrometers or more. These particles fragment during the production process and they finally constitute a small fraction of the finished product.

Little is known about the details of the fragmentation process and the particle morphology even though the manufacturing process is affected in an important way. Initial investigations with synchrotron computed microtomography (CMT) have shown that a silicasupported catalyst used for the polymerization of ethylene in the gas phase may have silica fragments distributed around the periphery of the particle [1]. In another investigation [2] it has been shown that a $\mathrm{MgCl}_{2}$-supported catalyst used for the polymerization of ethylene in a heptane slurry fragments more uniformly and that there is no evidence for a concentration of catalyst near the polymer surface. The polymer particles that are formed in a slurry are found to be more porous than those made in a gas phase.

In order to optimize the process variables, systematic studies are necessary. The use of tomographic sections is valuable from both a quantitative and a qualitative standpoint. In this paper it is pointed out that the use of histograms showing the distribution of measured linear attenuation coefficients is a simple approach to obtaining a measure of the particle porosity. Further, it is emphasized that an autocorrelation analysis also gives the porosity, and, in addition, a measure of the specific surface area and pore size distribution on a microscopic scale. These determinations should complement those obtained with the macroscopic methods of liquid nitrogen adsorption and mercury porosimetry since they do not entail assumptions about the connectivity of the pore structure. 


\section{EXPERIMENTAL METHODS}

The following catalysts were chosen for investigation in this experiment: a silicasupported catalyst used in the gas phase and a $\mathrm{MgCl}_{2}$-supported catalyst used in a slurry. It was known from previous experiments that the former produced a low porosity polyethylene pellet and the latter a high porosity pellet.

The analysis was carried out using the NSLS X26 x-ray microscopy facility. The details of this apparatus have been given elsewhere [3,4]. Briefly, the beam is collimated to a few micrometers size using tantalum slits. A collimated scintillation detector is used to measure the attenuation of the $\mathbf{x}$-ray beam in the specimen. The tomogram is then obtained using a first generation CT data collection technique. The beam is scanned across the material while the transmitted radiation is measured with a $\mathrm{CaF}_{2}$ detector using a sampling interval of a few micrometers. Between each translation the sample is rotated through a small angle. The tomogram is then reconstructed from the resulting data.

\section{AUTOCORRELATION ANALYSIS}

The n-point correlation function

$$
\chi_{n}\left(\vec{r}_{1}, \vec{r}_{2}, \ldots, \vec{r}_{n-1}\right)=\left\langle p(\vec{x}), p\left(\vec{x}+\vec{r}_{1}\right), \ldots, p\left(\vec{x}+\vec{r}_{n-1}\right)\right\rangle
$$

is the probability that the $n$-points separated by the spatial vectors $r_{1}, r_{2}, \ldots, r_{n-1}$ have the same value for the property $\mathrm{p}(\mathrm{x})$. The brackets $<>$ indicate a spatial average over the variable $\mathrm{x}$. For example, if $\mathrm{p}$ represents phase type, then for a binary medium of two phases, solid and void, the $\mathrm{n}$-point correlation function measures the probability that $\mathrm{n}$ such spatial locations lie, on the average, in the same phase. If the medium is isotropic, then the correlation function depends only on the relative orientation of the vectors $r$ with respect to each other. If the medium is stationary, correlation functions performed on samples of the medium from different spatial locations will be identical. 
Limiting discussion to the phase type, then for an isotropic bi-phase material, where $p=1$ for the void phase and $p=0$ for the solid phase, the porosity $\phi$ of a medium sample is given by

$$
\phi-\chi_{1}-\chi_{2}(|\vec{r}|-0)
$$

Further, if the phase structure contains no long range order

$$
\lim _{|\vec{r}| \rightarrow \infty} \chi_{2}(|\vec{r}|)-\phi^{2}
$$

Variations in local porosity can be determined by computing $\chi_{1}$ on medium samples from a variety of spatial locations. For a stationary medium, the porosity is constant and $x_{1}$ need only be determined for a single sample. Further, if the medium is isotropic, the porosity $\phi$ can be determined by a one dimensional computation for $\chi_{1}$, assuming the sample is long enough in one of its dimensions.

To illustrate the connection between the statistical correlation functions and medium properties, Berryman [6] concentrated on porosity and permeability in rocks. There is, of course, no problem in applying the same analysis to the case of the polyethylene polymerization particles. Walsh and Brace [7], used a cylindrical tube pore model to relate rock permeability $\mathbf{k}$ (assumptions are isotropic, stationary medium) to porosity, electrical formation factor $F$, and specific surface area $s$,

$$
k=\phi^{2} / \mathrm{bFs}^{2},
$$

where, we have seen, $\phi$ can be obtained from the 1-point correlation function, and $\mathrm{s}$ can be obtained from the 2-point correlation function slope at zero distance

$$
s=-4 \frac{\partial \chi_{2}(r)}{\partial r} I_{0}
$$


In the Walsh-Brace formula, $\mathrm{b}$ is a measure of the tube cross section in the model. This empirical parameter provides an effective tube cross section which can be determined or adjusted for various rock types or for other materials such as catalysts.

Berryman and Milton [8] have a rigcrous bound on the rock permeability based upon a variational principle first applied to porous media by Prager [9].

$$
k \leq \frac{2}{3} \int_{-}^{0} d r \frac{\chi_{2}(r)-\phi^{2}}{[1-\phi]^{2}}
$$

Berryman [6] has developed methods for the efficient evaluation of the 2- and 3-point correlation functions for planar images assuming medium isotropy in the plane. Porosity and specific surface area can be measured from the correlation function and an estimate of mean particle size can be inferred. It should be noted that 2-point correlation functions can be rapidly evaluated using Fourier transform techniques. Berryman's approach has been used in the evaluation of the catalyst tomograms presented here.

\section{RESULTS AND DISCUSSION}

The size and uniformity of $x$-ray absorption in the unused silica catalyst support particles are displayed in the tomogram shown in fig. 1A. The pixel size in the figure is 2 $\mu \mathrm{m} \times 2 \mu \mathrm{m}$ with a slice thickness of $2 \mu \mathrm{m}$. An expanded view of a single particle is given in fig. 1B. A small fissure can be seen that is roughly $6 \mu \mathrm{m}$ in width. This demonstrates the high resolution of the $\mathrm{x}$-ray tomography apparatus.

The fragmentation of the silica particles when used to polymerize ethylene is evident in the tomogram shown in fig. 2. Here, the tomogram is a section through a polyethylene particle which was obtained at a polymer yield of $200 \mathrm{~g}$ polymer per gram of catalyst. The pixel size was $3 \mu \mathrm{m} \times 3 \mu \mathrm{m}$ and the slice thickness was $3 \mu \mathrm{m}$. The bright areas near the 
periphery of the specimen denote the presence of silica fragments, several with an area larger than that of a single pixel.

A histogram showing the frequency distribution of the reconstructed attenuation coefficients for the same particle is shown in fig. 3. Only the region in the interior of the particle is included in the analysis. Only a single peak is visible, demonstrating that the porosity of the particle is very low. The noise in the tomogram limits the ability to resolve contributions from pixels that represent pores from those that represent polyethylene. In this case a limit could be put on porosity or alternatively, use of the cor-elation function can reveal the same information (see below).

A tomogram for the polyethylene $\mathrm{MgCl}_{2}$-supported catalyst particle is shown in fig. 4 . The difference in porosity compared to the silica-supported catalyst is evident. The attenuation coefficient histogram is shown in fig. 5. The large area in the image occupied by the pores is shown by the large peak centered at $0 \mathrm{~m}^{-1}$. A comparison of the peak areas gives a value of 0.33 for the porosity.

The results of the two-point correlation analysis for the two tomograms shown in figs. 2 and 4 are displayed in figs. 6 and 7. The main problem in carrying out the correlation function analysis results from the overlapping of the two peaks representing the pore and polymer fractions. The analysis requires unique identification of each pixel as belonging to either sample or pore space. Thus a lower pixel value (discriminator level) must be determined, below which all intensity values are assumed to belong to pore pixels, and above which sample pixels are assumed. Hence the results of the porosity and the specific surface area measurements will depend on the discriminator level used.

The discriminator level is less critical in the case of the silica catalyst since the porosity is low. Here we determine a porosity of 0.017 and a specific surface area of $0.0094 \mu \mathrm{m}^{-1}$. Assuming the material density is $1 \mathrm{~g} / \mathrm{cm}^{3}$, this corresponds to a surface area per unit mass of $0.0094 \mathrm{~m}^{2} / \mathrm{g}$. Using a reasonable choice for the discriminator level, the results for the 
$\mathrm{MgCl}_{2}$ catalyst are a porosity of 0.412 and a specific surface area of $0.110 \mu \mathrm{m}^{-1}$ or a surface area per unit mass of $0.110 \mathrm{~m}^{2} / \mathrm{g}$. Both the computed porosity and specific surface area in this second case are more susceptible to the discriminator level; it is difficult to determine whether the values determined are an overestimation or underestimation given the specific choice of level.

In both samples, the specific area calculation is obtained from a finite-difference calculation of the slope of the correlation function at zero distance. This slope was computed using the correlation data at distances of 0 and 1 pixel widths. For the first sample the pixel width is 5.0 micrometers, and 4.0 micrometers in the second. This finite pixel size and the corresponding slope determination tends to give an underestimate of the specific surface area.

These results can be compared with values obtained by conventional method of mercury porosimetry. Koffi et al. [2] report specific surface area values for the silica based material of $0.23 \mathrm{~m}^{2} / \mathrm{g}$ and for the $\mathrm{MgCl}_{2}$ material of $0.26-0.82 \mathrm{~m}^{2} / \mathrm{g}$. Our correlation function determined values are lower for the silica material and in reasonable agreement for the $\mathrm{MgCl}_{2}$

Differences can arise from

- the finite pixel size, mentioned above,

- the specific choice of discriminator level

- the fact that tomography makes it possible to measure porosity and surface areas in pores that are not connected to the surface of the particle. This is not possible with traditional techniques.

- CMT gives images of particular sections through the particle. Since the pore distribution is not necessarily uniform, deviations are not surprising. 
Koffi et al. [2j also report an average pore size of $30-40 \mu \mathrm{m}$ for catalysts with a yield of $75-80 \mathrm{~g} / \mathrm{g}$. The correlation functions do not show any strong evidence for structure in this size range.

\section{CONCLUSIONS}

It has been shown that the combination of synchrotron-based computed microtomography with the n-point correlation analysis gives a means of measuring the microscopic porosity and specific surface area of polyethylene polymerization particles. There is evidence that the values obtained in this way are consistent with those obtained using conventional porosimetry. Extension of the CMT measurements to multiple slices through the pellets will make it possible 10 study the fragmentation and transport of the catalyst in the polymer/monomer mixture and define particle porosity and surface area with much greater sophistication than is now possible and thus give improved insights into the production process. The success of the method rests on the use of the high brilliance synchrotron radiation $\mathrm{x}$-ray source and a careful development of the $\mathrm{x}$-ray tomography system.

These results are characteristic for the specific sample systems that were studied. We have found that the morphology of the catalyst/polymer particle depends on the specific conditions (temperature, pressure, pretreatment, etc.) of the polymerization as much as they do on the phase in which the polymerizations were condlict?d. Thus, we do not wish to generalize the results that we have described to represent the differences between gas and slurry processes or silica versus $\mathrm{MgCl}_{2}$ as supports. Our research reveals that the morphology of the growing polymer/catalyst is a mplex function of both compcsition and polymerization conditions. 


\section{REFERENCES}

[1] W. C. Conner, S. W. Webb, P. Spanne, and K. W. Jones, Macromolecules 23 (1990) 4742.

[2] E. Koffi, M. A. Ferrero, R. Sommer, and W. C. Conner, to be published.

[3] P. Spanne and M. L. Rivers, Nucl. Instrum. Methods B24/25 (1987) 1063; P. Spanne and M. L. Rivers, BioScience Abs. 1 (1988) 101.

[4] W. C. Conner, R. Sommer, M. Ferrero, K. Jones, and P. Spanne, to be presented at American Institute of Chemical Engineers Meeting, Los Angeles, CA, Nov. 1991; W. C. Conner, S. Webb, R. Sommer, M. Ferrero, E. Koffi, K. Jones, and P. Spanne, to be presented at American Institute of Chemical Engineers Meeting, Los Angeles, CA, Nov. 1991.

[5] S. Torquato and G. Stell, J. Chem. Phys. 77 (1974) 2071.

[6] J. G. Berryman, J. Appl. Phys. 57 (1985) 2374.

[7] J. B. Walsh and W. F. Brace, J. Geophys. Res. (1984).

[8] J. G. Berryman and G. W. Milton, J. Chem. Phys. 83 (1985) 754.

[9] S. Prager, Phys. Fluids 4 (1961) 1477. 


\section{FIGURE CAPTIONS}

Fig. 1A. Tomogram showing sections through a number of typical $\mathrm{SiO}_{2}$ catalyst support particles. The volume element examined in the section was $8 \mu \mathrm{m}^{3}$.

Fig. 1B. Magnified view of a single particle chosen from those shown in fig. 1A. The average width of the crack in the particle is about $5 \mu \mathrm{m}$.

Fig. 2. Tomogram showing a section through a polyethylene polymerization particle produced using a $\mathrm{SiO}_{2}$-supported $\mathrm{Cr}$ catalyst. The polymer yield is $200 \mathrm{~g}$ polymer per $g$ catalyst. The catalyst fragments are distributed at the periphery of the section.

Fig. 3. Histogram showing the distribution of linear attenuation coefficients within the polymer particle tomogram shown in fig. 2. The large peak represents the polyethylene. Events can be found at higher attenuation coefficients are the catalyst fragments, but are not visible on this scale. The absence of events at 0 attenuation coefricient shows that the porosity of the material is low.

Fig. 4. Tomogram showing a section through a polyethylene polymerization particle produced with a $\mathrm{MgCl}_{2}$-supported Ti catalyst. The polymer yield is $80 \mathrm{~g}$ polymer per $\mathbf{g}$ catalyst. The catalyst is distributed throughout the particle in the specimen. The high porosity of the specimen is clearly seen.

Fig. 5. Histogram showing the distribution of linear attenuation coefficients within the polymer particle tomogram shown in fig. 4. The two peaks show the distribution of voids and polyethylene in the specimen and, in contrast to the tomogram shown in fig. 2 , that the porosity is high.

Fig. 6. Autocorrelation function for the tomogram shown in fig. 2.

Fig. 7. Autocorrelation function for the tomogram shown in fig. 4. 


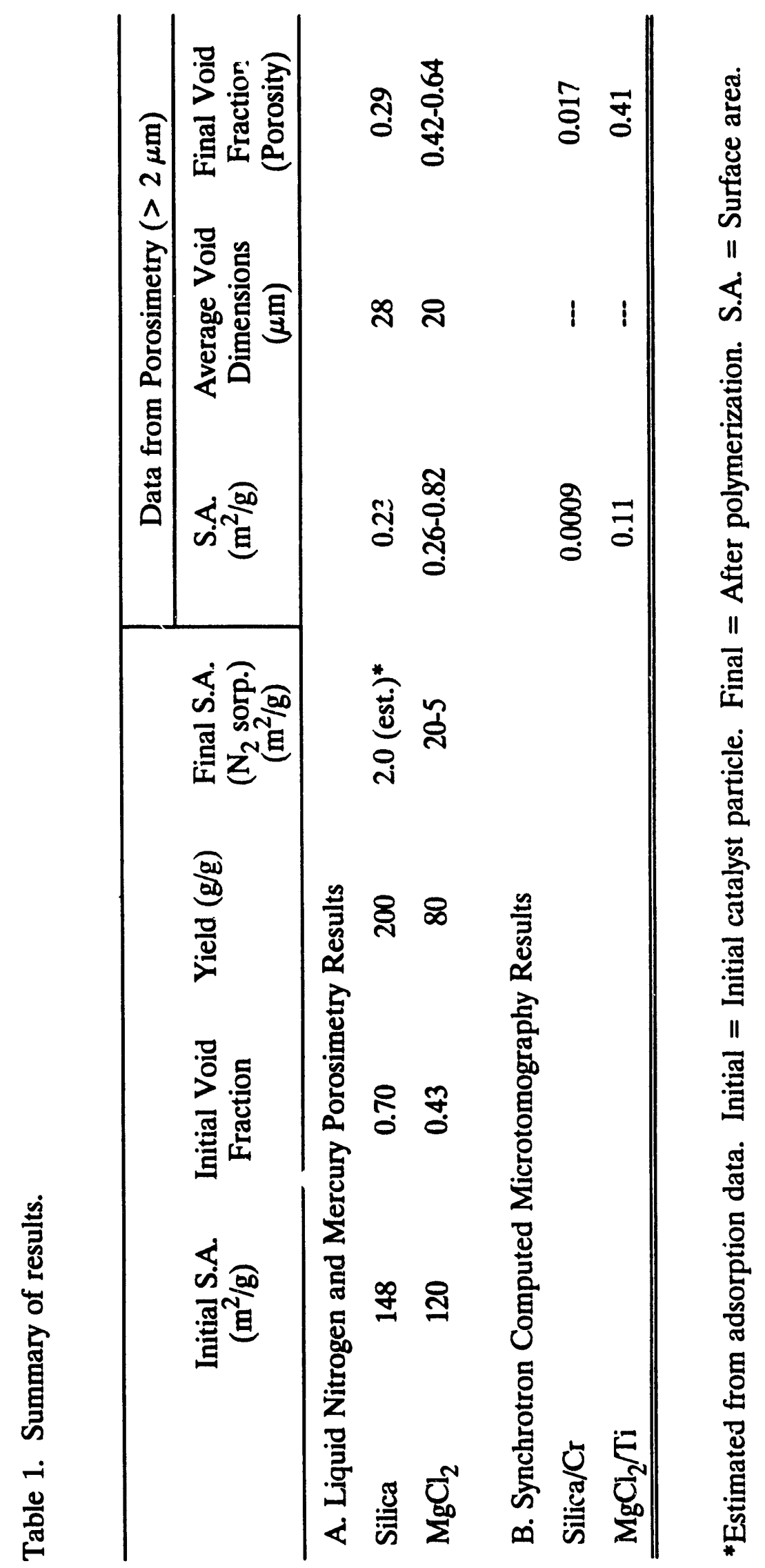



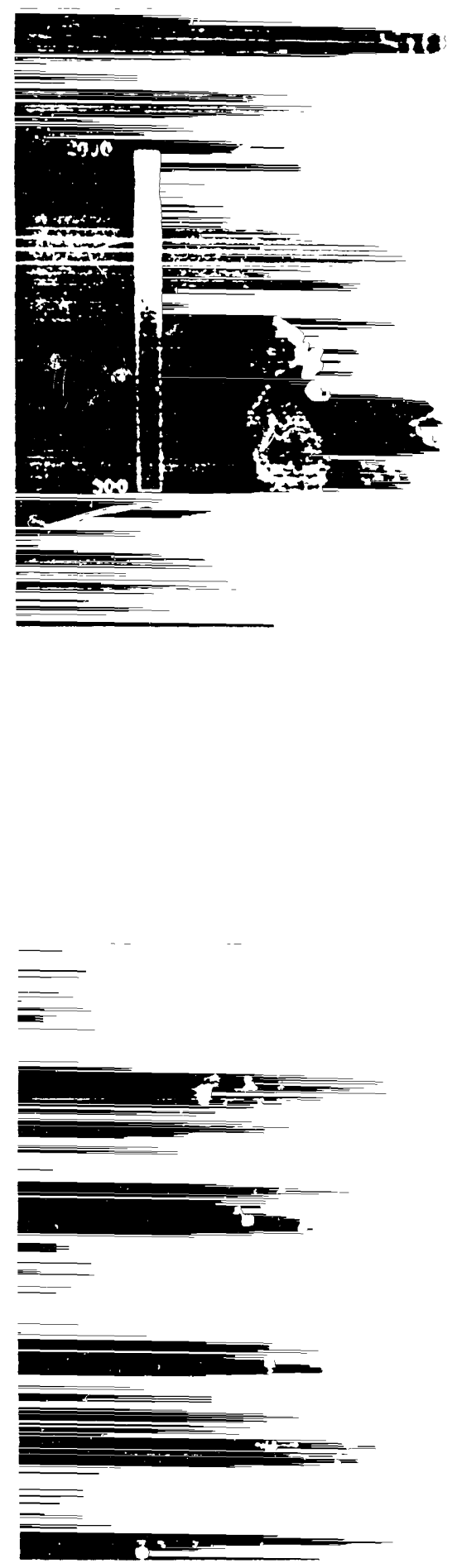


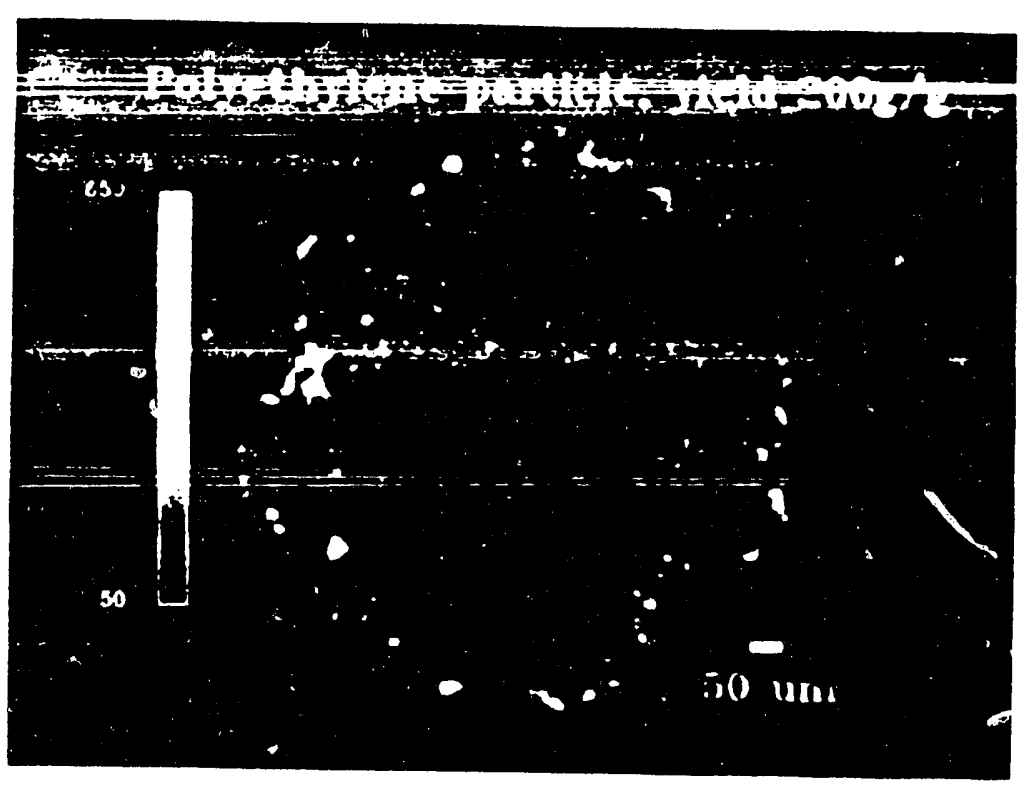

Fig. 2 


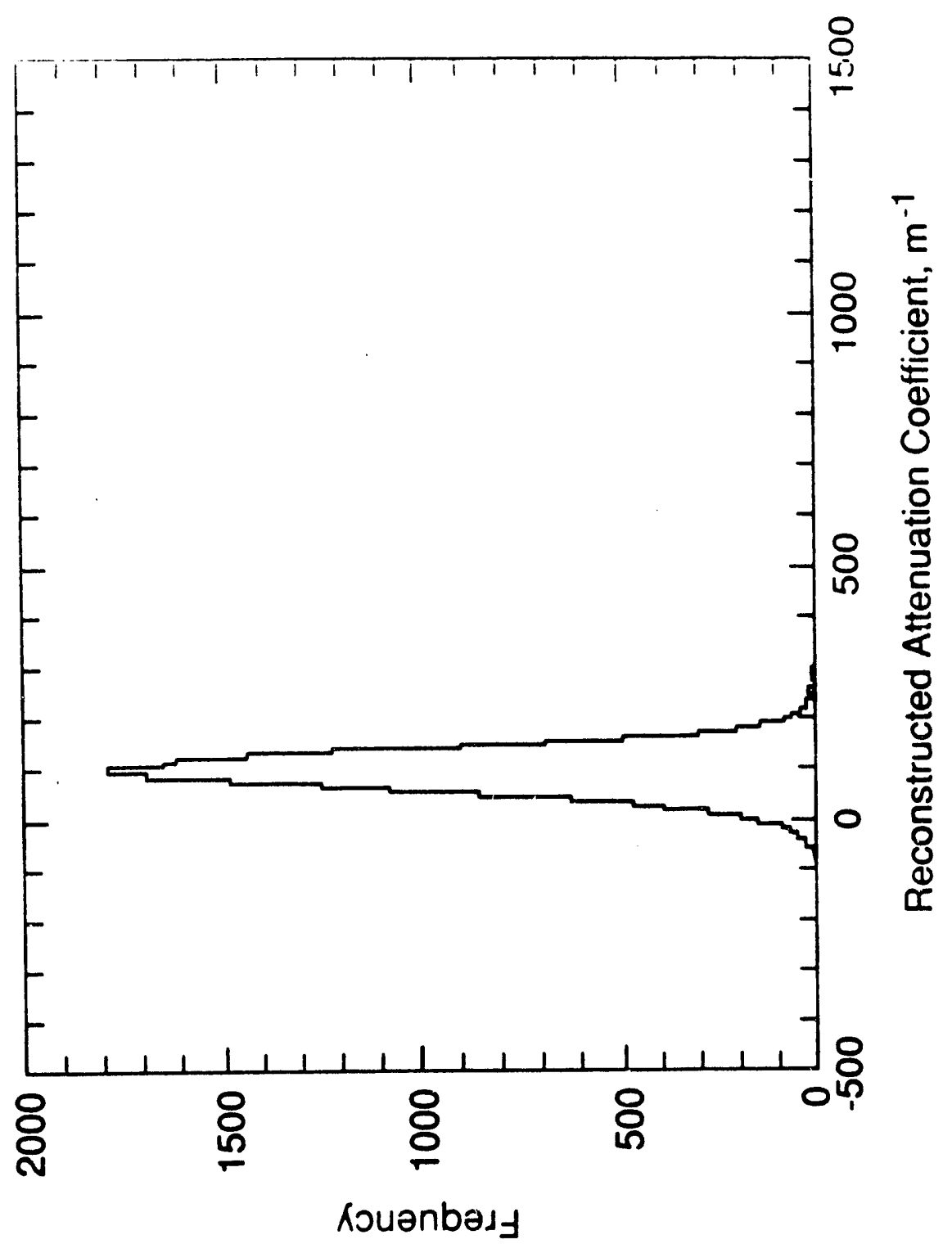




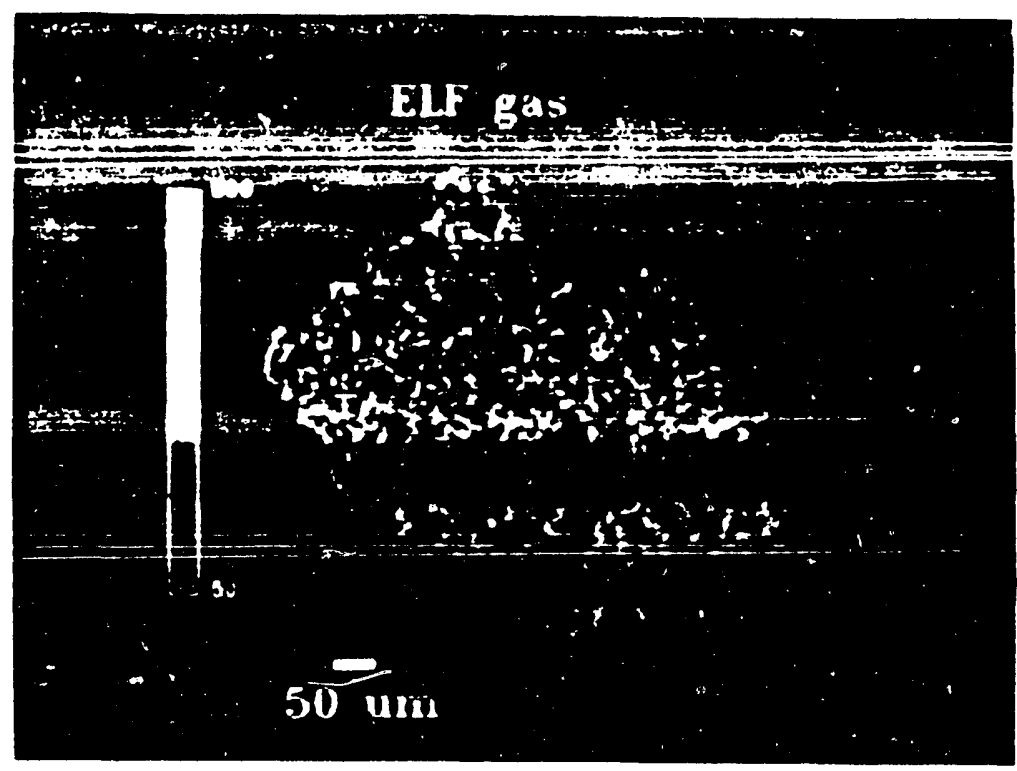

Fig. 4 


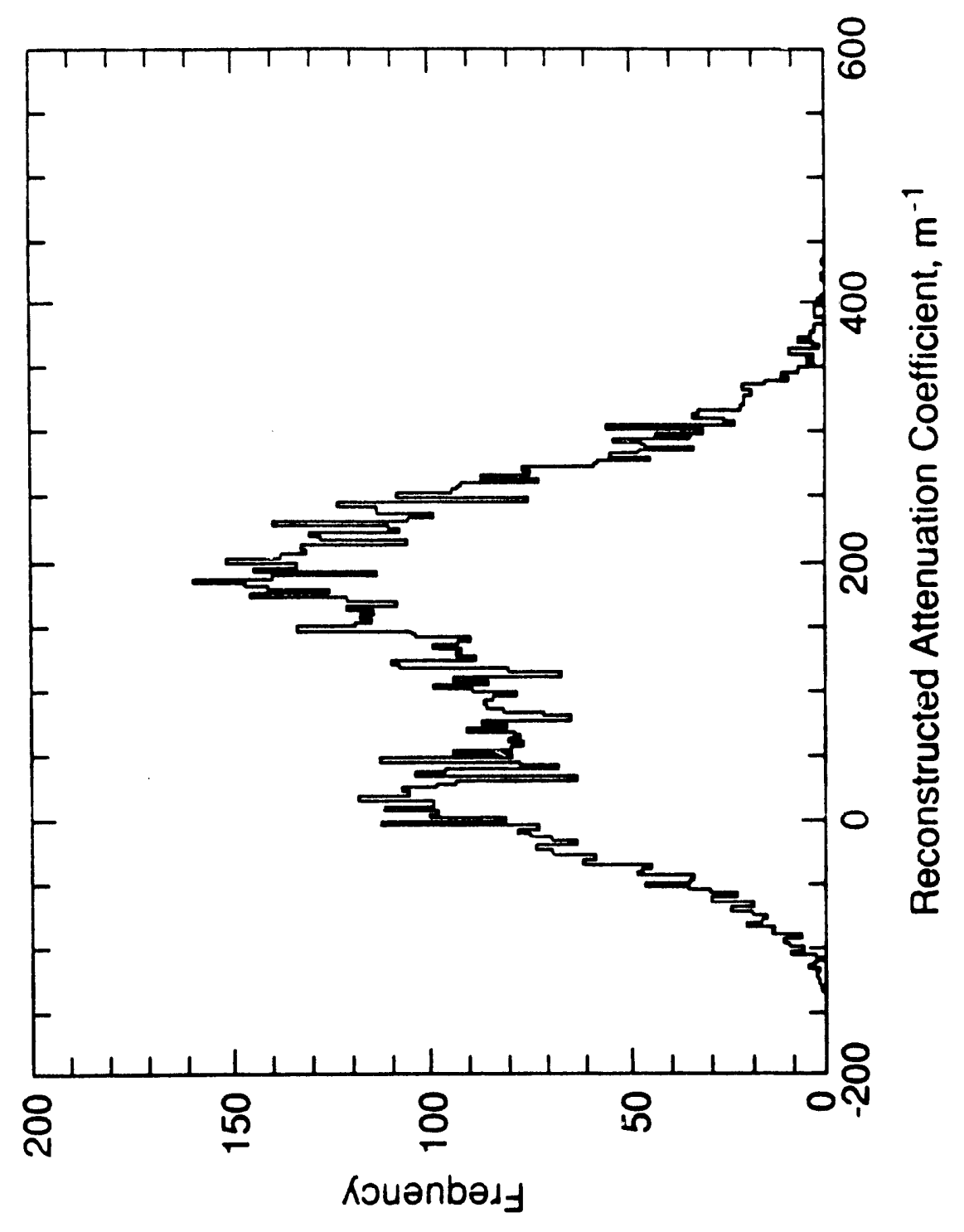




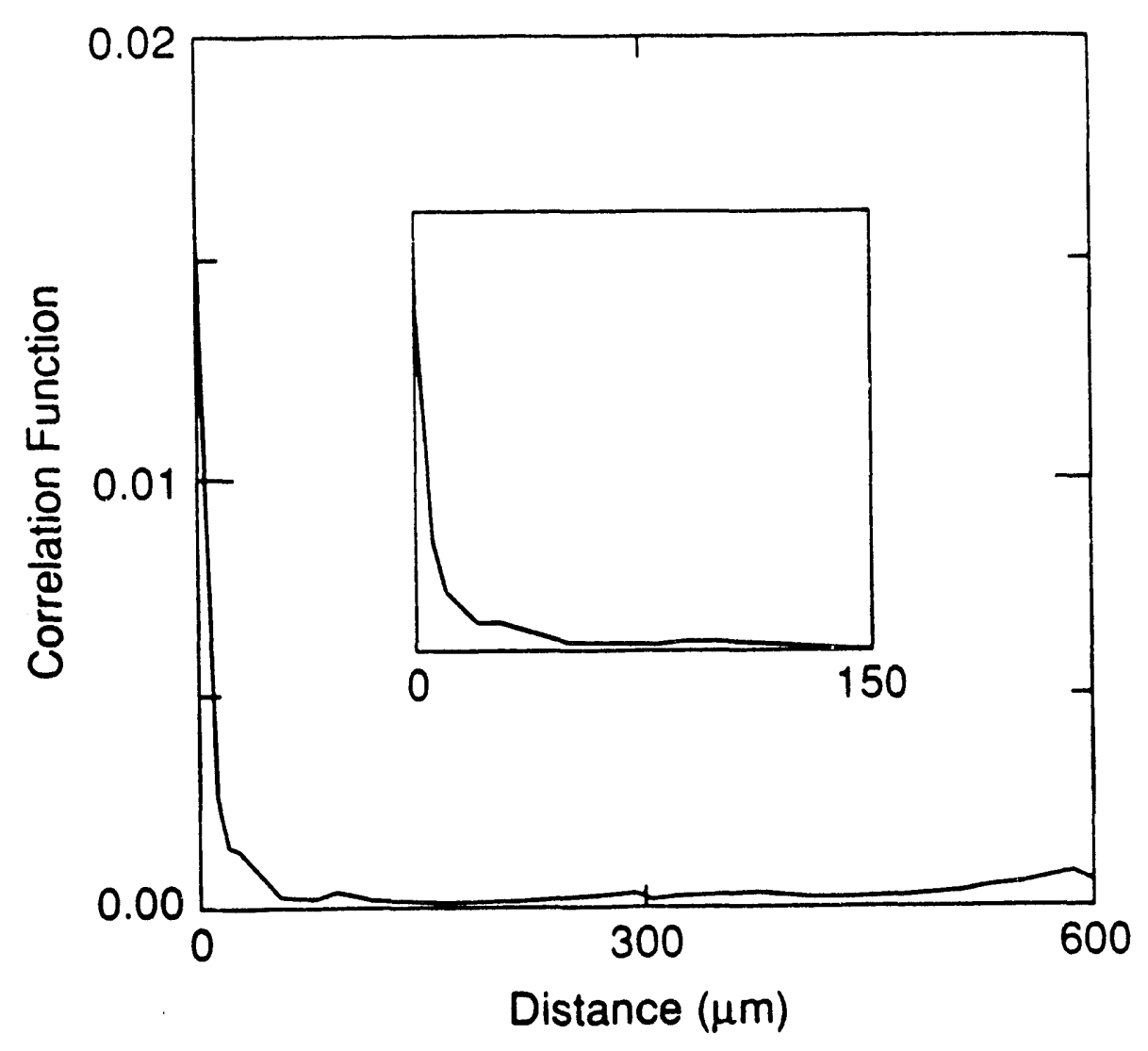

Fig. 6 


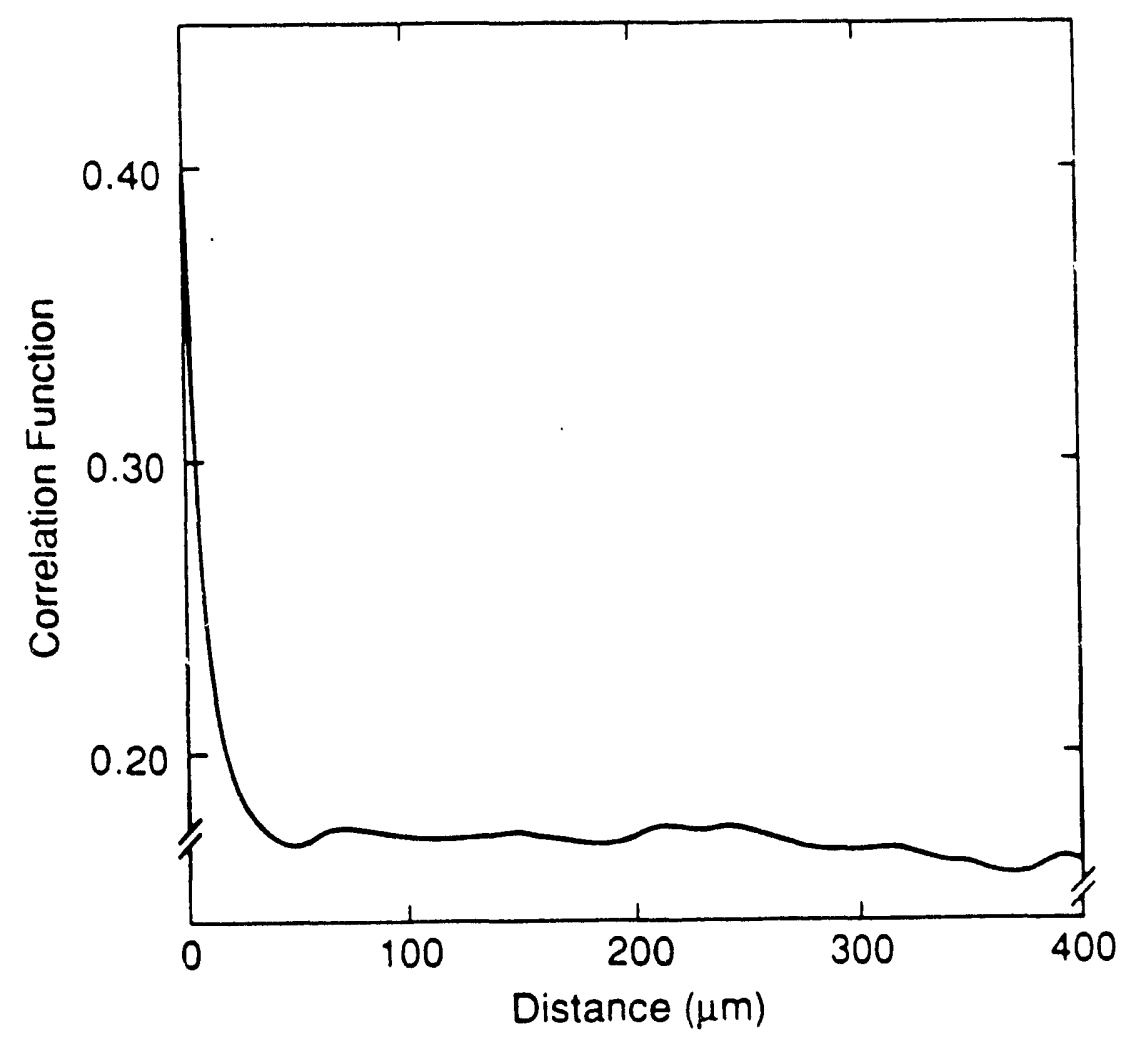

Fig. : Jones la $3:: ;$ 


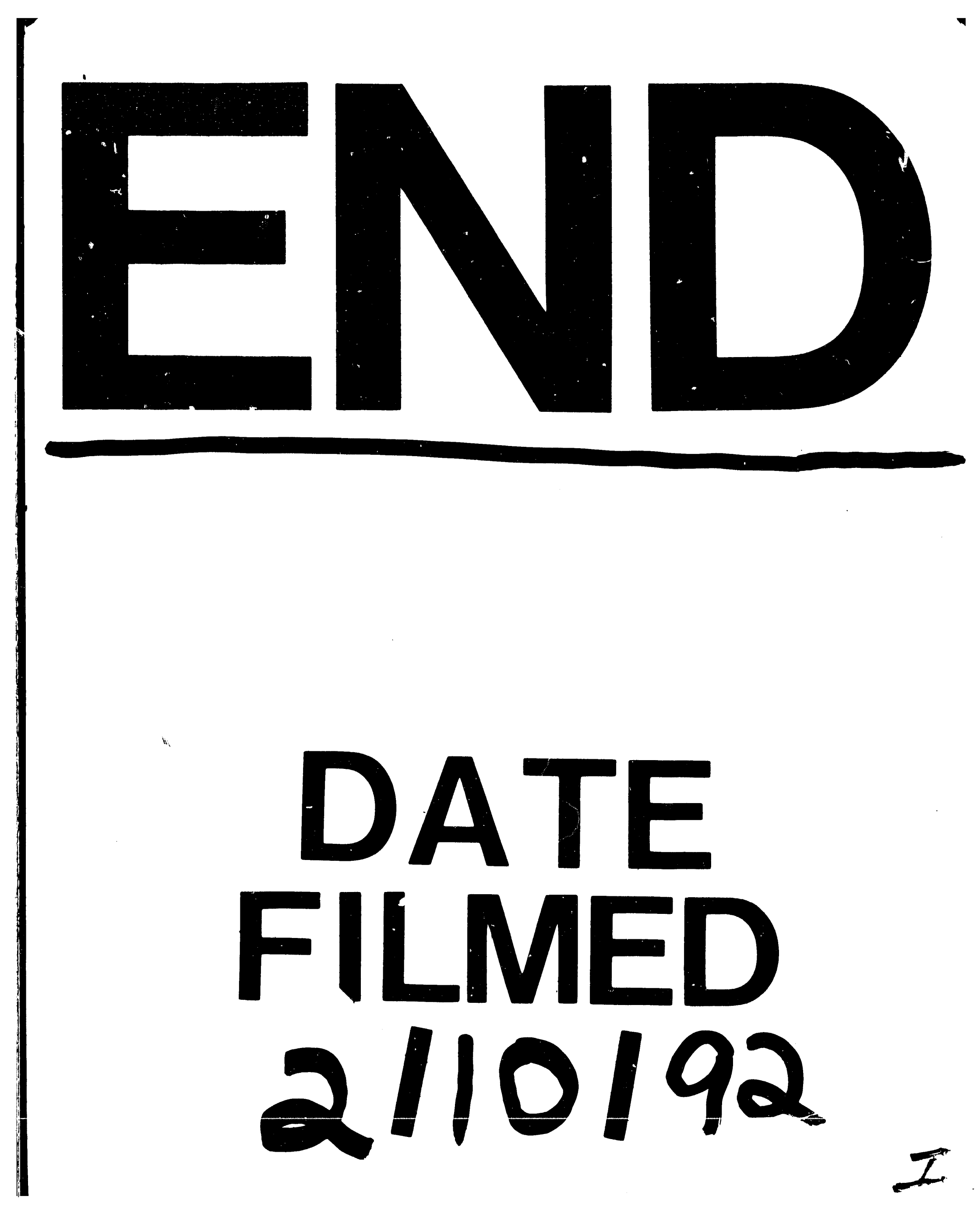


